\title{
Article
}

\section{Personalized Prediction of Postconcussive Working Memory Decline: A Feasibility Study}

\author{
Yung-Chieh Chen ${ }^{1,2 *}$, Yung- Li Chen ${ }^{3 *}$, Duen-Pang Kuo ${ }^{1,2}$, Yi-Tien Li 1,4,*, Yung-Hsiao Chiang 4,5,6,7, Jyh-Jong Chang \\ 3 , Sung-Hui Tseng 8,9, Cheng-Yu Chen 1,2,10,11
}

1. Translational Imaging Research Center, Taipei Medical University Hospital, Taipei 110, Taiwan; $\underline{173002 @ \text { h.tmu.edu.tw }}$ (Y.-C. C.); $194029 @$ h.tmu.edu.tw $($ D.-P. K.); angela810727@tmu.edu.tw (Y.-T. L.); sandy0932@tmu.edu.tw (C.-Y.C.)

2. Department of Medical Imaging, Taipei Medical University Hospital, Taipei 110, Taiwan

3. Department of Occupational Therapy, Kaohsiung Medical University, Kaohsiung 807, Taiwan; u107706003@kmu.edu.tw (Y.-L. C.); jichang@kmu.edu.tw (J.-J. C.)

4. Neuroscience Research Center, Taipei Medical University, Taipei 110, Taiwan; ychiang@tmu.edu.tw (Y.-H C.)

5. Center for Neurotrauma and Neuroregeneration, Taipei Medical University, Taipei 110, Taiwan

6. Department of Neurosurgery, Taipei Medical University Hospital, Taipei 110, Taiwan

7. Department of Surgery, School of Medicine, College of Medicine, Taipei Medical University, Taipei 110, Taiwan

8. Department of Physical Medicine and Rehabilitation, Taipei Medical University Hospital, Taipei 110, Taiwan; d301091012@tmu.edu.tw (S.-H. T.)

9. Department of Physical Medicine and Rehabilitation, School of Medicine, College of Medicine, Taipei Medical University, Taipei 110, Taiwan

10. Department of Radiology, School of Medicine, College of Medicine, Taipei Medical University, Taipei 110, Taiwan

11. Research Center for Artificial Intelligence in Medicine, Taipei Medical University, Taipei 110, Taiwan

* These authors contributed equally to this work.

* Correspondence: angela810727@tmu.edu.tw (Y.-T.L.)

Tel.: +886-2-2736-1661\#8900

\begin{abstract}
:
Concussion, also known as mild traumatic brain injury (mTBI), commonly causes transient neurocognitive symptoms, but in some cases, it causes cognitive impairment, including working memory (WM) deficit, which can be long-lasting and impede a patient's return to work. The predictors of long-term cognitive outcomes following mTBI remain unclear because abnormality is often absent in structural imaging findings. The purpose of the study was to determine whether machine learning-based models using functional magnetic resonance imaging (fMRI) biomarkers and demographic or neuropsychological measures at baseline could effectively predict 1-year cognitive outcomes of concussion. We conducted a prospective, observational study of patients with mTBI who were compared with demographically-matched healthy controls enrolled between September 2015 to August 2020. Baseline assessments were collected within the first week of injury, and follow-ups were conducted at 6 weeks, 3 months, 6 months, and 1 year. Potential demographic, neuropsychological, and fMRI features were selected according to the significance of correlation with the estimated changes in WM ability. The support vector machine classifier was trained using these potential features and estimated changes in WM between the predefined time periods. Patients demonstrated significant cognitive recovery at the third month, followed by worsened performance after 6 months, which persisted until 1 year after concussion. Approximately half of the patients experienced prolonged cognitive impairment at 1-year follow up. Satisfactory predictions were achieved for patients whose WM function did not recover at 3 months (accuracy=87.5\%), 6 months (accuracy $=83.3 \%$ ), 1 year (accuracy=83.3\%), and performed worse at 1 -year follow-up compared to baseline assessment (accuracy=83.3\%). This study demonstrated the feasibility of personalized prediction for long-term postconcussive WM outcomes based on baseline fMRI and demographic features, opening a new avenue for early rehabilitation intervention in selected individuals with possible poor long-term cognitive outcomes.
\end{abstract}


Keywords: concussion; mild traumatic brain injury; working memory; long-term cognitive outcome; support vector machine classifier; personalized prediction

\section{Introduction}

Mild traumatic brain injury (mTBI), commonly referred to as concussion, typically does not present with visual findings on structural magnetic resonance imaging (MRI) examinations, and therefore, providing neuroimaging evidence to support diagnosis or therapeutic evaluation is difficult. Furthermore, mTBI can cause an array of postconcussive symptoms (PCS), most notably headache, sleep deficit, fatigue, dizziness, depression, anxiety, and cognitive impairment [1]. The average time required for symptom relief in most individuals is approximately 3 months [2]; however, some individuals with subjective PCS continue to experience symptoms even 1 year after concussion [3, 4]. Postconcussive neuropsychological deficits have been suggested to be secondary to cognitive deficits [5-7]. Studies have suggested that only $15 \%$ of first-time concussed individuals continue to experience persistent neuropsychological symptoms [8, 9]; however, approximately half of them experience long-term cognitive impairment that persists for years and can severely affect overall quality of life [10,11]. The options for early treatment of mTBI remain rather limited due to a general lack of validated biomarkers with a high degree of sensitivity and specificity for the development of symptom-specific therapies. Thus, useful clinical biomarkers for individualized postconcussive management must be urgently identified, particularly to target individuals with poor long-term cognitive outcomes.

Working memory (WM) involves the ability to transiently store and manipulate information to be used for cognitive or behavioral activities. WM deficit is one of the most common postconcussive cognitive impairments [12]. Chen et al. demonstrated reduced activation in the regions of $N$-back WM circuitry in patients with mTBI during both moderate and high WM load conditions compared with healthy controls (HCs), especially prominent under WM 2-back > 1-back condition [13]. Differences were identified in WM functional activity between both patients with symptomatic mTBI and HCs as well as patients' baseline assessment and 6-week follow-up, whereas no difference was observed in neuropsychological and behavioral performance, including digit span score, continuous performance test and WM task performance, suggesting that the deficits in WM functional activity estimated from functional magnetic resonance imaging (fMRI) may have a higher sensitivity to mTBI than to neuropsychological and behavioral evaluations alone [13].

We hypothesized that long-term cognitive outcomes of mTBI can better be predicted using pooled fMRI, demographic, and neuropsychological biomarkers than by using neuropsychological evaluations alone. In this prospective observational study, our objectives were to identify fMRI, demographic, or neuropsychological biomarkers at baseline that could best predict future cognitive changes during the year following a concussion and to construct machine learning-based predictive models to discriminate between patients at high risk of poor long-term cognitive outcomes and patients with normal recovery. Specifically, the $N$-back WM task ( $N=1$ and 2$)$ were performed to obtain potential diseaserelated fMRI features since WM 2-back > 1-back condition could show the most prominent changes of impaired WM circuitry after mTBI [13]. Additionally, machine learning algorithms can unravel the relationship between input variables (e.g., biomarkers) and response variables (e.g., cognitive outcome) through a data-learning process, which allows for the prediction of future cognitive changes for each individual or the stratification of a patient population based on characteristic features. Furthermore, understanding various potential biomarkers associated with postconcussive WM impairments may render it possible to translate these biomarkers into effective cognitive rehabilitation strategies to improve, or at least mitigate impediments to, the recovery of WM function, which is important in most occupations. 
At present, for cognitive rehabilitation management, detailed holistic neuropsychiatric assessments are required to identify, establish, and develop adaptive general or domain-specific interventions, whether adopting a nonpharmacologic or pharmacologic approach [14]; this is particularly true for the treatment of posttraumatic deficits in memory and executive function, as different compensatory training strategies are applied based on impairment severity [15]. Our aim was to construct a framework for precise individualized prediction of postconcussive cognitive outcomes based on the early fMRI and neuropsychological biomarkers assessed at baseline to facilitate early therapeutic intervention and individualized rehabilitation strategies.

\section{Materials and Methods}

\subsection{Participants and neuropsychological evaluation}

Between September 2015 and August 2020, 70 right-handed patients with mTBI (age $=37.9 \pm 12.2$ years; 23 [32.9\%] women) and 48 age-, sex-, and education-matched righthanded HCs (age $=37.4 \pm 12.0$ years; 16 [33.3\%] women) consented to participate in the study at Taipei Medical University Hospital. Reasons for injury were as follows: motor vehicle accident $(n=37)$, fall $(n=17)$, sports $(n=3)$, assault $(n=8)$, and other $(n=5)$. Patients were followed up at 6 weeks ( $n=34 ; 52.52 \pm 6.95$ days), 3 months ( $=29 ; 100.96 \pm 13.56$ days), 6 months ( $n=28 ; 195.95 \pm 14.61$ days), and 1 year $(n=25 ; 376.48 \pm 16.52$ days) after concussion. In total, 24 patients (38.6\%) completed the baseline and all five follow-up sessions. Patients dropped out during follow-up visits mainly because of failure to keep in touch through phone calls or e-mails or a change in residence or job. All participants had normal or corrected-to-normal visual activity and no history of neurological or psychiatric disorders. This study was approved by the Institutional Review Board of Taipei Medical University Hospital before data collection (TMUH TMU-JIRB No. 201504083, N201612008, N201904032, and N202102008) and conducted according to the original and amended Declaration of Helsinki.

The following operational definition of mTBI was used in the current study: patients with closed-head injury manifesting in a loss of consciousness lasting for $<30$ min, initial Glasgow Coma Scale score $>13$, and normal findings in computed tomography of the entire brain. The exclusion criteria were prior neuropsychiatric illnesses or symptoms, brain injury history, any coexisting or previous neurological illnesses, and contraindication for MRI. Inclusion criteria for the control group were the same, except for a negative assessment for mTBI and no concussion history.

Neuropsychological assessments, namely six types of clinical symptom measures, the Mini-Mental State Examination (MMSE), and the Wechsler Adult Intelligence Scale, fourth edition (WAIS-IV), were conducted by a clinical psychologist on the same day as the initial and follow-up MRI scans. The clinical symptoms were assessed using the Glasgow Outcome Scale-Extended (GOSE), Pittsburgh sleep quality index, Epworth Sleepiness Scale, Dizziness Handicap Inventory, Rivermead Post Concussion Symptoms Questionnaire (RPQ), Beck Anxiety Inventory, and Beck Depression Inventory, for which higher scores indicate greater symptomatology. Moreover, WM ability was assessed using the WM index (WMI), arithmetic ability (AMT), and digit span score (DS).

\subsection{MRI data acquisition and experimental design}

MRI data were obtained using a 3T MRI scanner (Siemens MAGNETOM Prisma, Erlangen, Germany), and a 64-channel head coil was used to acquire the fMRI time series. Standard single-shot gradient-echo echo planar imaging-based fMRI (TR/TE = 2000/20 ms, flip angle $=90^{\circ}$, voxel size $=3 \times 3 \times 3.5 \mathrm{~mm} 3$, matrix $=64 \times 64 \times 40$, and 105 volumes) was performed. Participants were instructed to keep their eyes closed and not entertain any particular thoughts while remaining awake, alert, and as motionless as possible.

The experimental design of the $N$-back task in fMRI was performed using Presentation software (Version 18.1, Neurobehavioral Systems, Inc., Berkeley, CA, USA) and is presented in Figure S1. An N-back task contains three epochs, each composed of a 30- 
second task period and a 30-second fixation on a crosshair. The interstimulus interval between each trial during a task period was 2 seconds. In total, 45 trials were performed, with each task consisting of $80 \%$ nontarget trials and $20 \%$ target trials. In each run of the $\mathrm{N}$-back tasks, participants were instructed to pay attention to a series of six-digit numerical stimuli and respond by using the right index finger to press the button whenever the current stimulus matched the number that had been presented $N$ times previously $(N=1$ or 2) [16].

For the coregistration and normalization of fMRI data, three-dimensional T1weighted magnetization-prepared rapid gradient-echo images (TR/TE/TI = 2300/3.26/1030 ms, flip angle $=8^{\circ}$, voxel size $=1 \times 1 \times 1 \mathrm{~mm} 3$, and matrix $\left.=256 \times 256 \times 176\right)$ were obtained .

\subsection{Data analysis}

\subsection{1. fMRI preprocessing}

The anatomical and fMRI data were preprocessed using Statistical Parametric Mapping (SPM12; Wellcome Department, University College London, UK) for slice timing correction, realignment, spatial normalization to MNI space, and spatial smoothing with a 5mm full-width-at-half-maximum Gaussian kernel. Furthermore, linear and quadratic trends of fMRI time series were removed.

\subsubsection{WM task activation and deactivation map}

To calculate the brain activation and deactivation map during the N-back WM task, the experimental paradigm used the convolved canonical hemodynamic response function as a regressor in a general linear model. Six head-motion parameters estimated through image realignment by using SPM12 were used as covariates and partially regressed out of the preprocessed fMRI time series. A contrast image corresponding to the main effects of task performance was created and represented brain activity relative to the implicit baseline of unmodeled variance [17]. Group level activation and deactivation maps were then calculated as a one-sample t-test across all participants within each group.

\subsection{Statistical analyses}

A one-sample t-test was used to determine the significance within the HC or mTBI group, and a two-tailed two-sample t-test was used to observe between-group differences. A two-tailed paired-sample t-test was conducted to examine the significance between the initial and follow-up data. The statistical tests were corrected for multiple comparisons by controlling the false discovery rate (FDR) to $q=0.05$ to avoid errors related to multiple comparisons in these calculations.

\subsection{Regions-of-interest selection and percentage signal change calculation}

The regions-of-interest (ROIs; Table S1) were first defined using a 3-mm-diameter sphere centered at the WM 2-back task activation and deactivation peak regions in the HC group ( $p<0.01$, FDR corrected; Figure 1). The percentage signal change map [18] of each participant's WM condition was then estimated through multiplication of the regression coefficient map for the main effects of task performance approximated as the quotient by dividing the peak value by the constant term in the design matrix such that they could be compared across participants [17]. Finally, the percentage signal change value of each participant's WM condition at each ROI was then extracted. 

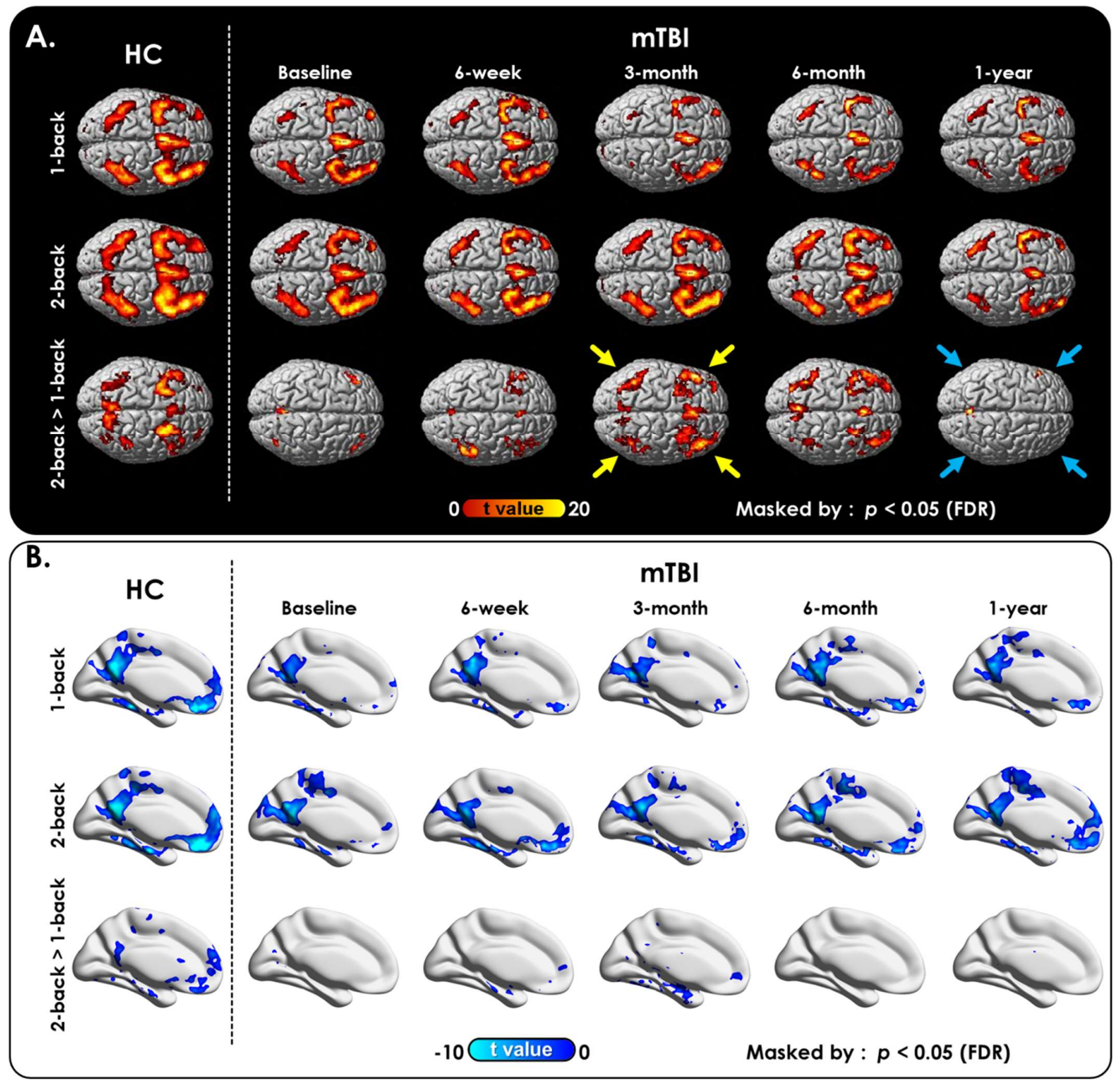

Figure 1. Postconcussive working memory activation and deactivation changes over time between baseline and follow-up. (A) Activation and (B) deactivation maps of 1-back, 2-back, and 2-back > 1-back WM conditions in HCs and patients with mTBI at each time point. Patients showed significant recovery under the WM 2-back > 1-back condition (bottom row) after 3 months (yellow arrows) but worsened again at 1-year follow-up (blue arrows). Note that the statistical tests were corrected for multiple comparisons by controlling the false discovery rate (FDR) to $q=0.05$ to avoid errors related to multiple comparisons in these calculations.

\subsection{Postconcussive WM changes at predetermined time periods during 1-year follow-up}

To determine the courses of postconcussive WM changes, we first retrospectively assessed the 24 patients who completed the baseline and all five follow-up sessions to determine the status of WM decline or recovery at each time point during the 1-year followup period. The two-tailed paired-sample t-test was applied to examine the significance 
between the baseline and follow-up data to identify the statistically significant progression of postconcussive cognitive decline at predetermined time points (periods). Specifically, these statistically significant time periods of cognitive changes were regarded as the meaningful time periods for the machine learning-based approach in terms of postconcussive cognitive progression prediction. Patients were further divided into "poor outcome" and "good outcome" groups according to the negative and positive slopes of cognitive changes within the specific time period.

\subsection{Individualized prediction of postconcussive WM impairments by using biomarkers measured at baseline}

The percentage signal change extracted from ROIs for the WM task performed by each participant was used for N-back WM fMRI features. Neuropsychological assessments (namely seven types of neuropsychological tests, clinical symptom measures, and the WAIS-IV test) and the demographic data (namely age, sex, education year, and score on the GOSE) were treated as the potential neuropsychological and demographic features, respectively. The candidate fMRI, demographic, and neuropsychological features were selected if there were a significant correlation with the estimated changes in WMI during the specific time period to train the support vector machine (SVM) classifier with k-fold cross-validation ( $\mathrm{k}=10$ in this study) for each prediction to achieve a reliable and unbiased estimate of machine learning model performance on a limited dataset sample [19]. Specifically, the complete data set is first divided into $\mathrm{k}$ consecutive folds. Then each fold is used once as a validation, and the remaining $\mathrm{k}-1$ folds form the training set. This approach may be computationally expensive, but it does not waste too much data (as is the case when fixing an arbitrary validation set), which is the main advantage in problems with very small sample size. The individual SVM classification approach was accomplished using in-house MATLAB (version R2020a, Mathworks, Sherborn, MA, USA) scripts.

\section{Results}

\subsection{Demographics}

In total, 70 patients with mTBI and $48 \mathrm{HCs}$ were recruited in this study (Figure S2). Among the patients, 24 who completed the baseline and all five follow-up sessions were selected for investigations regarding dynamic changes in cognitive functions after concussion. Table S2 lists the basic demographic characteristics of both groups. No significant between-group differences in terms of age, sex, and education were observed between patients and HCs. Furthermore, no significant within-group differences were observed in demographics between patients who completed the 1-year follow-up and those who completed only the baseline assessment following concussion. All structural MRI were unremarkable in terms of structural or signal changes.

\subsection{Postconcussive WM changes during 1-year follow-up period}

\subsection{1. $N$-back WM task}

In both groups, the $\mathrm{N}$-back WM task fMRI exhibited increased signals in the bilateral frontal and parietal lobes ( $p<0.01$, FDR corrected; Figure 1A), consistent with activation of WM circuitry. However, the extent of activation was less in the patient group than in HCs (Figure 1A, first two columns). Furthermore, the response of the brain to the increase in WM load from 1-back to 2-back, as shown in the brain activation (Figure 1A, bottom row) and deactivation (Error! Reference source not found.B) maps, was greater in HCs than in patients with mTBI. The WM deficit pattern of WM 2-back > 1-back and activation regions were recovered at the third month of follow-up; however, the WM deficit (decline) paradoxically reappeared again at 1-year follow-up after concussion. Lastly, deactivation in the patient group constantly disappeared at WM 2-back > 1-back activation regions throughout the 1-year follow-up. In addition, as the WM load increased from 1-back to 2- 
back, there would be an imbalance in the communication between task-positive (activation) and task-negative (deactivation) regions in the context of effortful task execution. Collectively, these findings revealed changes that persist during the chronic phase of mTBI and highlight the need for longitudinal studies to map the postconcussive cognitive decline and/or recovery.

\subsubsection{Neuropsychological assessment}

Assessment with the WAIS neuropsychological test revealed that WM-related abilities, as indicated by the WMI (Figure 2A), AMT (Figure 2B), and DS score (Figure 2C), significantly improved from 6 weeks to 3 months after mTBI but became worse again from 3 to 6 months after mTBI. The result matched with our fMRI results and provided converging evidence for the patients tend to have a transient cognitive recovery at the third month after mTBI, however, worsened again after 6 months. Because of the fluctuation and variation in the functional recovery of WM among patients, we grouped patients according to follow-up time periods, where the patients with recovered WMI were classified into "good outcome group" and the patients exhibited a decline in WMI were labeled as "poor outcome group" for prediction model analysis. The percentages of patients in the four predefined time periods were as follows:

1. $38 \%(9 / 24)$ of patients exhibited no recovery in WMI at 3 months after mTBI.

2. $75 \%(18 / 24)$ of patients exhibited a decline in WMI from 3 to 6 months after mTBI.

3. $38 \%(9 / 24)$ of patients exhibited no recovery in WMI from 6 months to 1 year after mTBI.

4. $46 \%(11 / 24)$ of patients exhibited worsened WMI at 1-year follow-up than at the baseline.
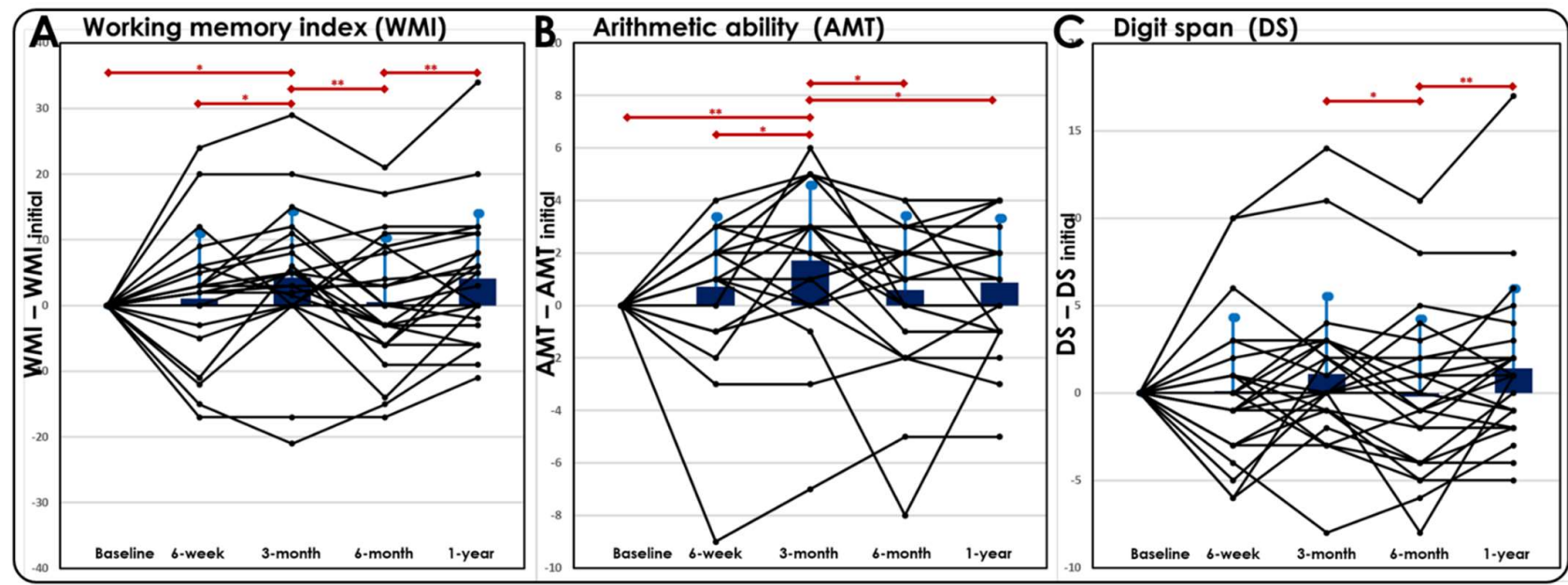

Figure 2. Postconcussive cognitive changes over time between baseline and follow-up. Dynamic individual patients' trajectories of (A) WMI, (B) AMT, and (C) DS at each time point. The trajectories were normalized by subtracting baseline measurements for better visualization. Patients exhibited significant recovery during the 3-month follow-up but worsened again from 3 months to 6 months or even at 1-year follow-up. Compared with baseline measurements, roughly half of the patients with mTBI displayed reduced cognitive function after 1 year.

\subsection{Prediction of postconcussive WMI decline based on baseline studies}

\subsubsection{WMI not recovered at 3 months after mTBI}

Figure 3A presents the importance weighting of features derived from candidate fMRI activation and deactivation patterns, demographics, and neuropsychological tests 
for predicting WMI changes between 6 weeks and 3 months after concussion. In particular, the selected features included two demographic (age and sex), five WM 1-back activation (left putamen, bilateral calcarine, left dorsolateral prefrontal cortex [dLPFC; BA46], and triangular part of the right inferior frontal gyrus ([IFG (tri.); BA45]), three WM 1-back deactivation (e.g., right middle cingulate cortex [MCC]), three WM 2-back activation (right dorsal anterior cingulate cortex [dACC], right rolandic operculum, and right inferior temporal gyrus [ITG]), and one WM 2-back deactivation (the left middle temporal gyrus [MTG]) features (Figure 3A,B); 87.5\% SVM prediction accuracy and an $82.96 \%$ area under the receiver operating characteristic curve (ROC-AUC; Figure 3C,D) were achieved using these features.

A.

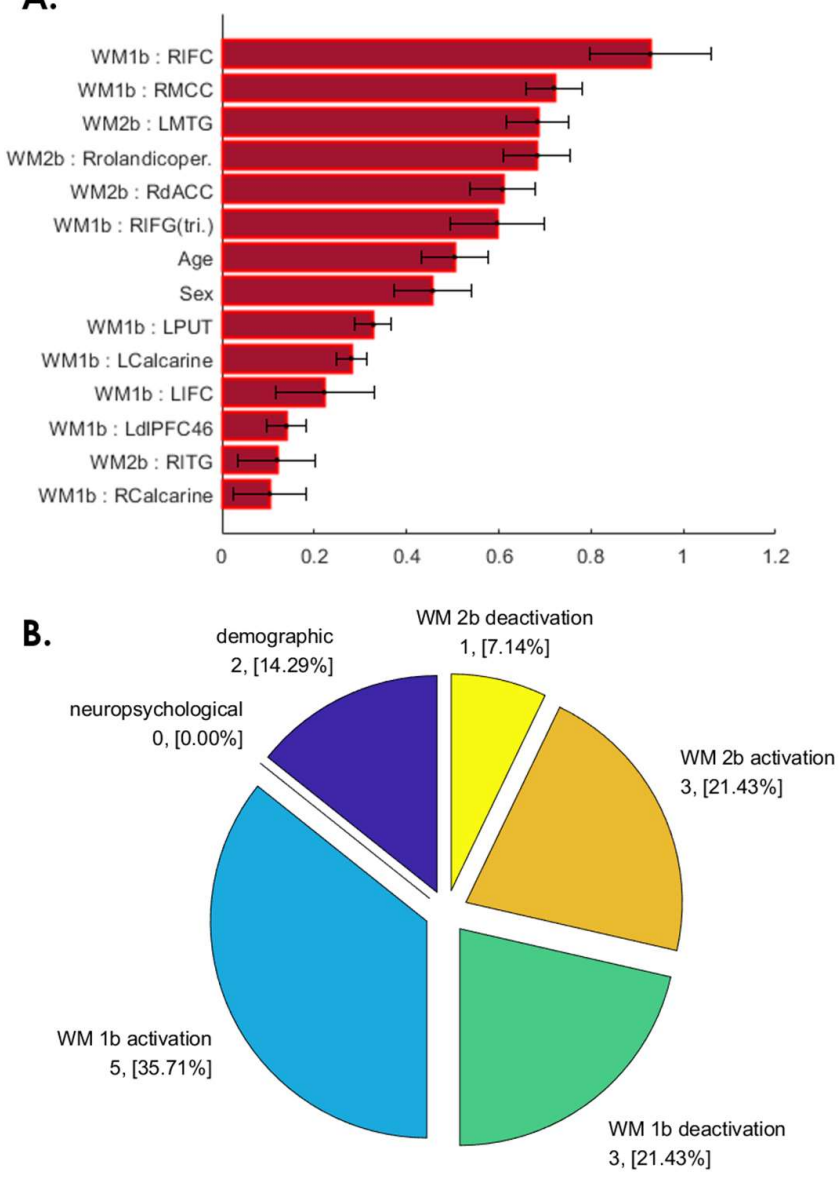

C.

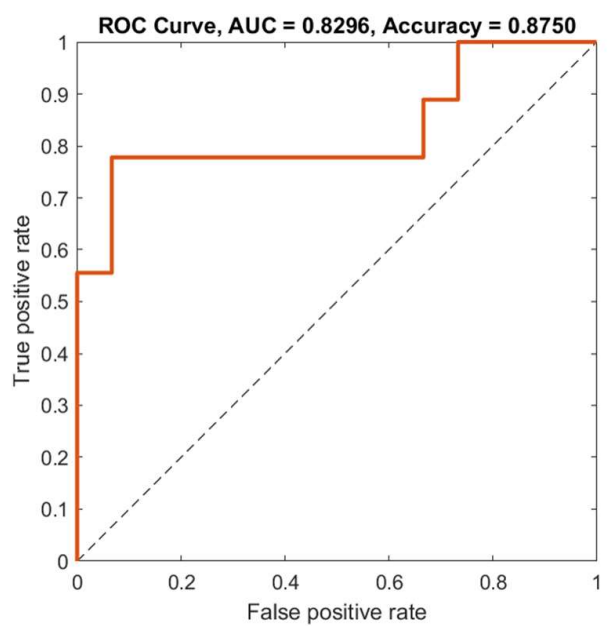

D.

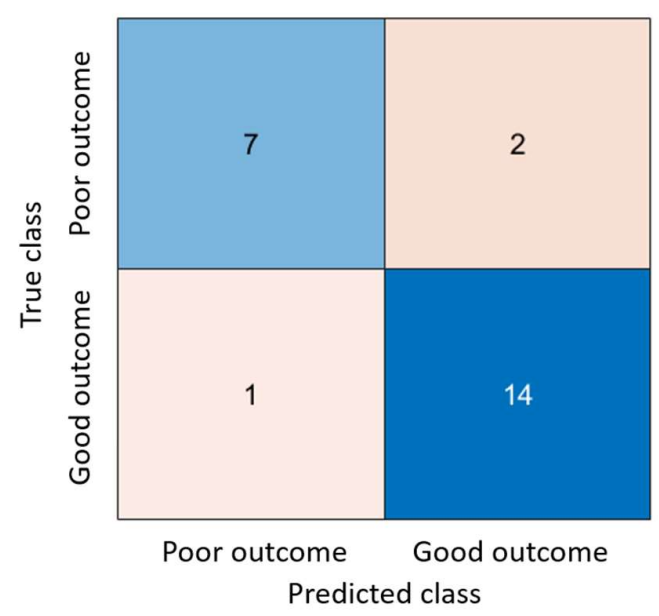

Figure 3. SVM predictive model for $\mathbf{3 7 . 5} \%$ of patients whose WM ability did not recover at 3-month follow-up. (A) The red bar graph and the corresponding error bar, respectively, represent the average and standard deviation of the discriminative feature weights among the 10 cross-validated SVM classifiers. (B) Profiles of selected features for constructing the SVM classification model. None of the neuropsychological features were selected for this predictive model. (C) ROC curve of the selected feature to differentiate the "poor outcome group" from the "good outcome group." (D) Confusion matrix to summarize the result of this binary classification model.

\subsubsection{WMI decline from 3 to 6 months after initial recovery}

Figure $4 \mathrm{~A}$ presents the importance weighting of features selected from among candidate fMRI results, demographics, and neuropsychological tests to predict WMI changes between 3 and 6 months after concussion. These features included three demographic 
(age, sex, and education years), one WM 1-back activation (left inferior frontal gyrus orbital part [IFG (orb.); BA46]), one WM 1-back deactivation (left MTG), two WM 2-back activation (e.g., bilateral IFG [tri.]), and two WM 2-back deactivation (left hippocampus and right MTG) features (Figure 4A,B). The SVM prediction accuracy could reach $83.33 \%$ with an $84.26 \%$ ROC-AUC (Figure 4C,D).

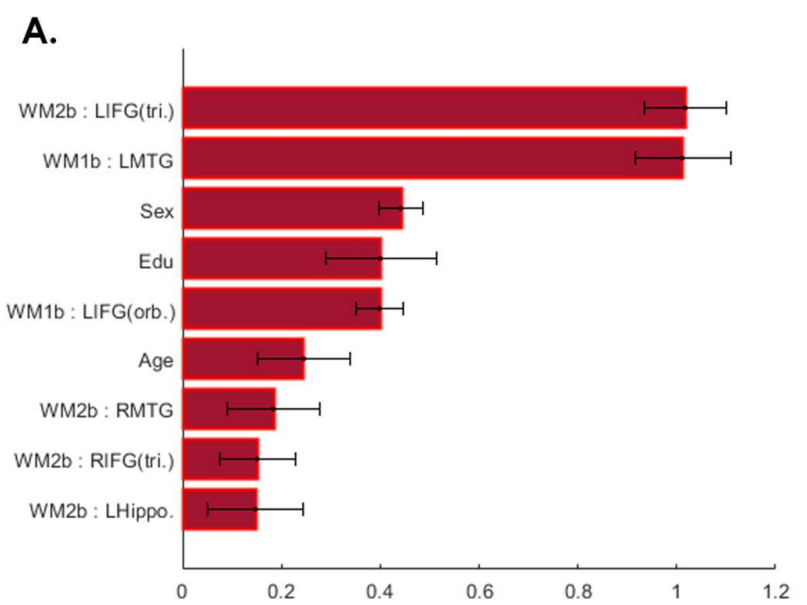

B.

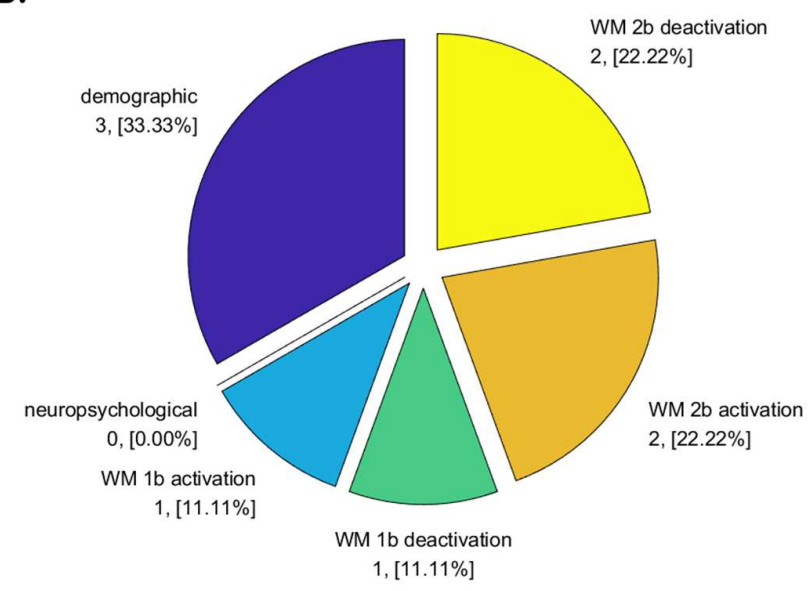

C.

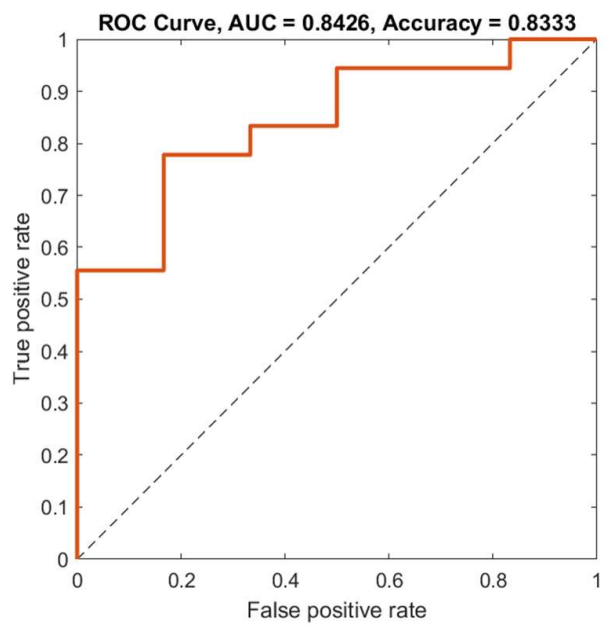

D.

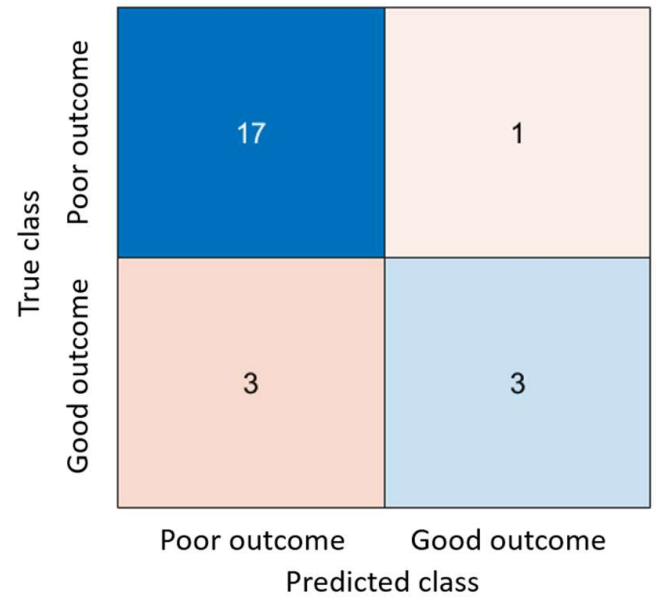

Figure 4. SVM predictive model for $75 \%$ of patients whose WM ability dropped from 3 to 6 months after concussion. (A) The red bar graph and the corresponding error bar, respectively, represent the average and standard deviation of the discriminative feature weights among the 10 cross-validated SVM classifiers. (B) Profiles of the selected features for constructing the SVM classification model. None of the neuropsychological features were selected for this predictive model. (C) ROC curve of the selected feature to discriminate the "poor outcome group" from the "good outcome group." (D) Confusion matrix to summarize the result of this binary classification model.

\subsubsection{WMI not recovered from 6 months to 1 year after mTBI}

Figure 5A shows the importance weighting of features selected from candidate fMRI results, demographics, and neuropsychological tests for predicting WMI changes between 6 months and 1 year after concussion. Features selected for SVM classification included 2 demographic features (age and sex), 10 WM 1-back activation features (bilateral anterior insula [AINS], bilateral IFG [orb.], bilateral putamen, bilateral rolandic operculum, right $\mathrm{dACC}$, and left inferior parietal sulcus [IPS]), 5 WM 1-back deactivation features (left posterior cingulate cortex [PCC], right angular gyrus, left hippocampus, left MTG, and left inferior frontal cortex [IFC]), 8 WM 2-back activation (bilateral IFG [orb.], bilateral AINS, bilateral ITG, left putamen, and right rolandic operculum), and 3 WM 2-back deactivation 
features (bilateral hippocampus and left amygdala; Figure 5A,B). With the use of the selected features, the SVM classifier could reach $83.33 \%$ accuracy with an $88.89 \%$ ROC-AUC (Figure 5C,D).

A.
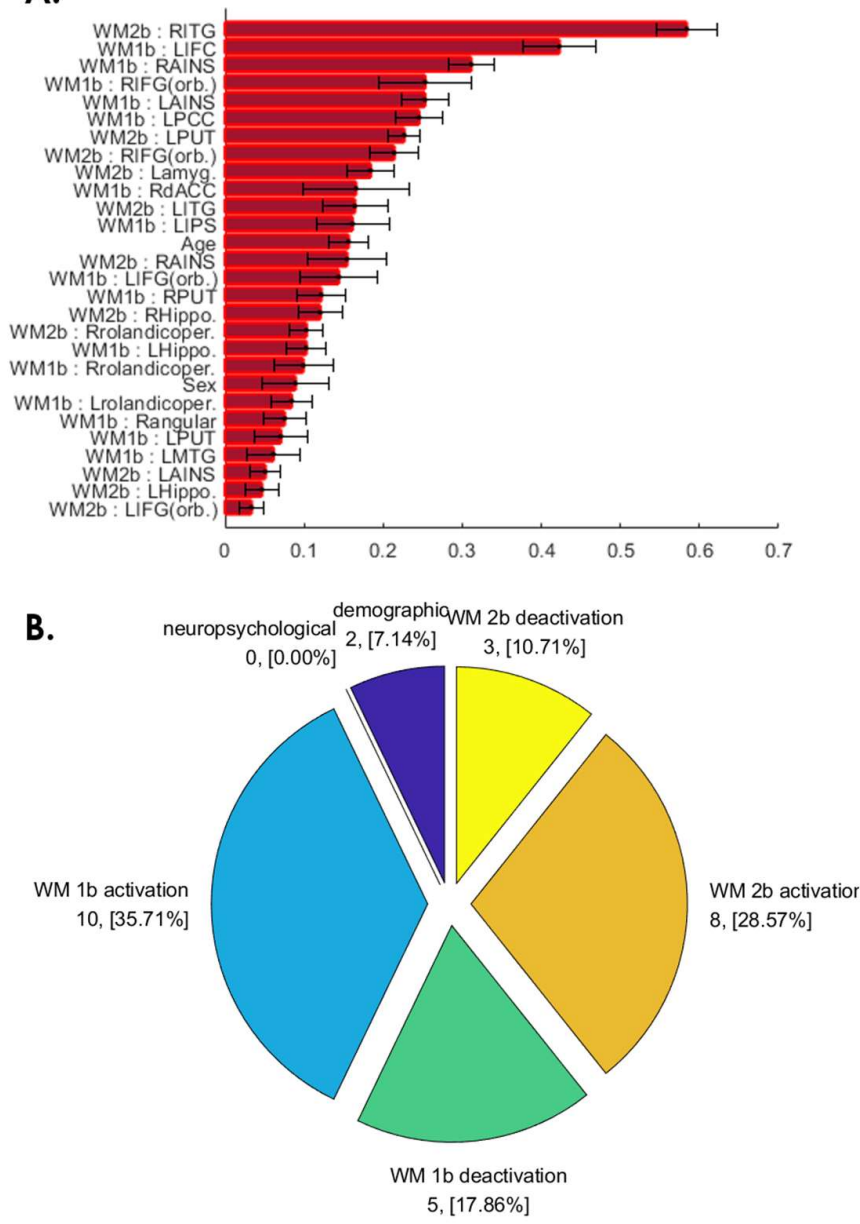

C.

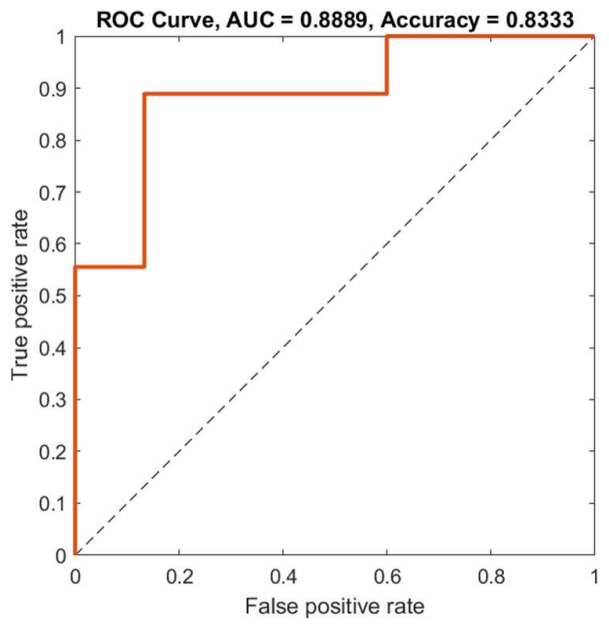

D.

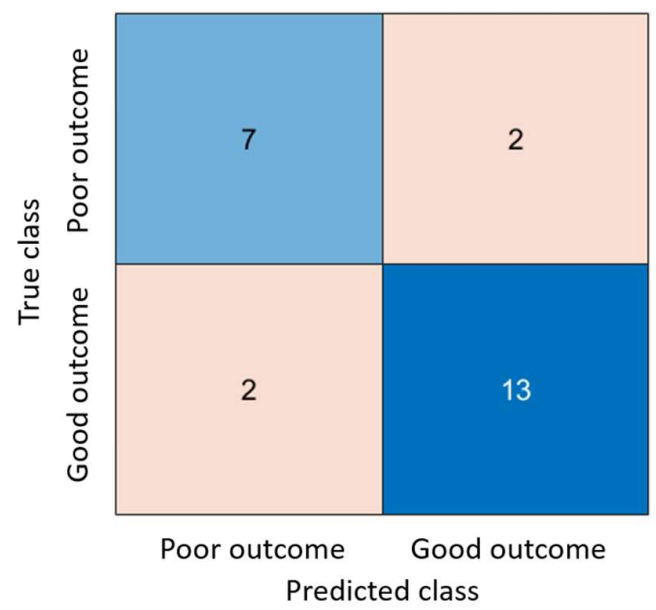

Figure 5. SVM predictive model for 37.5\% of patients whose WM ability did not recover from 6-month to 1-year follow-up. (A) The red bar graph and the corresponding error bar, respectively, represent the average and standard deviation of the discriminative feature weights among the 10 cross-validated SVM classifiers. (B) Profiles of the selected features for constructing the SVM classification model. None of the neuropsychological features were selected for this predictive model. (C) ROC curve of the selected feature to discriminate the "poor outcome group" from the "good outcome group." (D) Confusion matrix to summarize the result of this binary classification model.

\subsubsection{Patients whose WMI at 1-year follow-up was worse than at baseline}

The importance of the feature weighting derived from candidate fMRI, demographic, and neuropsychological features to predict the estimated changes of WMI between the baseline and 1-year after concussion are shown in Figure 6. Two demographic features (age and sex), four neuropsychological features (MMSE, DS, WMI, and AMT), two WM 1-back activation features (e.g., left ITG and left temporal parietal junction [TPJ]), two WM 1-back deactivation features (e.g., left ventromedial prefrontal cortex [vmPFC; BA25], left posterior insula [PINS]), and one WM 2-back deactivation feature (e.g., right hippocampus) were selected (Figure 6A,B), and the SVM reached $83.33 \%$ accuracy with a $95.80 \%$ ROC-AUC (Figure 6C,D). 
A.

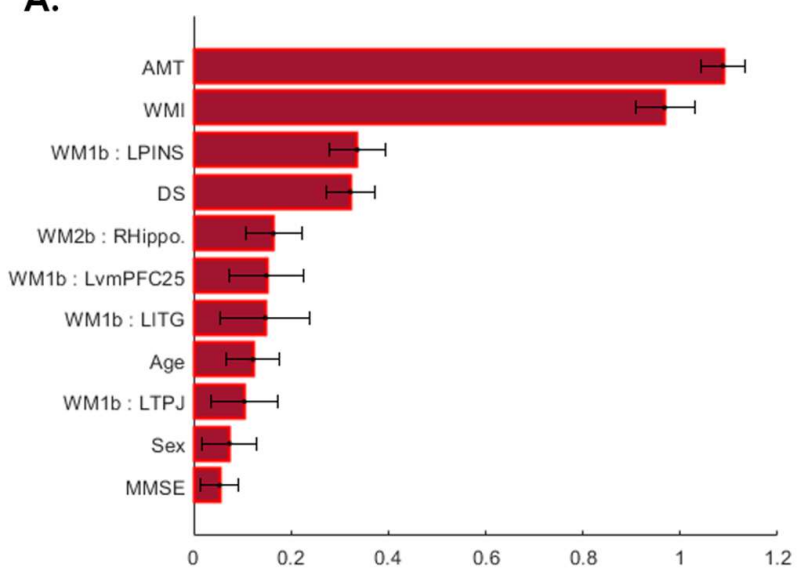

B.

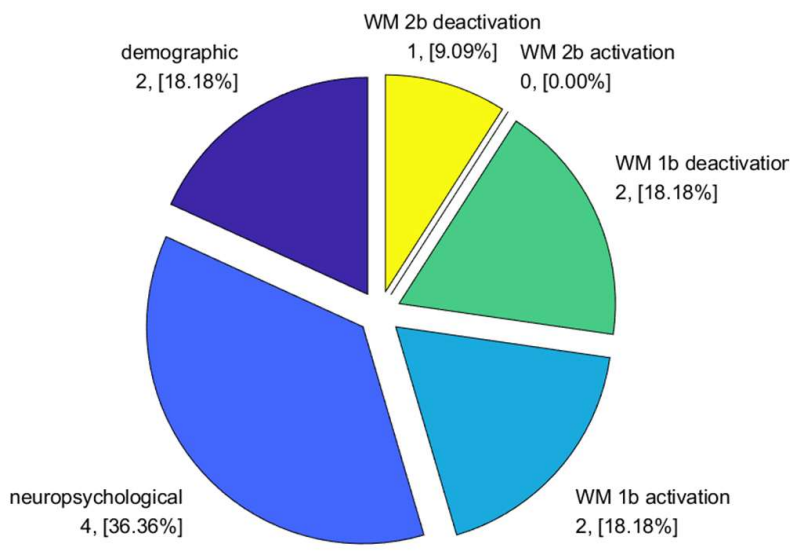

C.

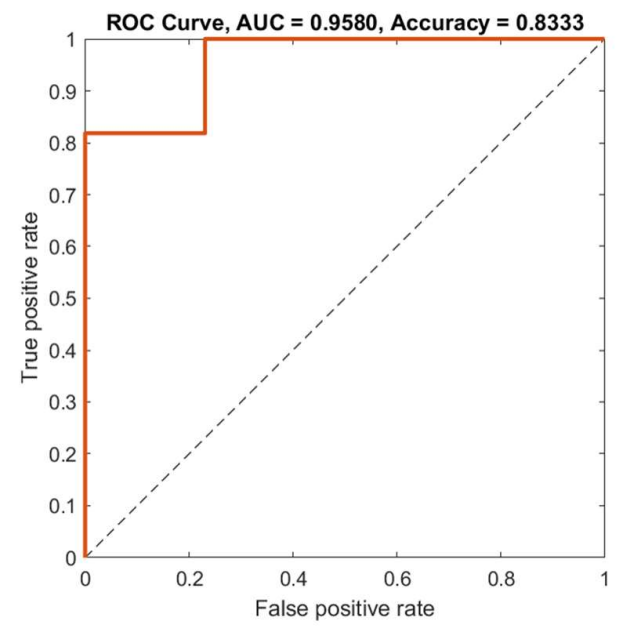

D.

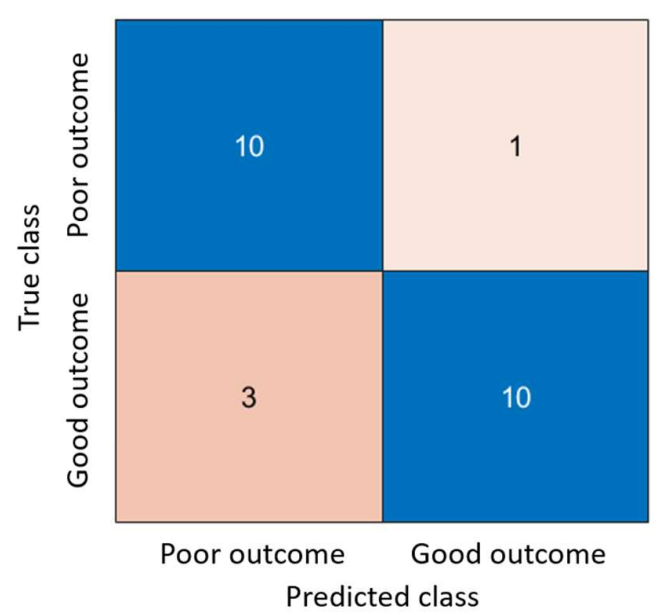

Figure 6. SVM predictive model for $45.83 \%$ of patients whose WM ability after 1 year became worse than at baseline. (A) The red bar graph and the corresponding error bar, respectively, represent the average and standard deviation of the discriminative feature weights among the 10 cross-validated SVM classifiers. (B) Profiles of the selected features for constructing the SVM classification model. None of the WM 2-back activation features were selected for this predictive model. (C) ROC curve of the selected feature to discriminate the "poor outcome group" from the "good outcome group." (D) Confusion matrix to summarize the result of this binary classification model.

\section{Discussion}

\subsection{Validate machine learning algorithms in a limited data size}

Previous studies that have aimed at predicting long-term postconcussive cognitive outcomes for mTBI have generally adopted a multivariate approach encompassing patient demographics, clinical symptoms, and neuropsychological features, as well as other factors such as health care utilization and premorbid psychiatric conditions [20, 21]. However, the performance of such predictive methods can be limited due to clinical variability and complexity, as well as confounding factors such as ambiguous documentation, undeclared medication use, and other concurrent medical conditions, and assessment of morphologic information based on structural brain imaging has not demonstrated additional benefits [22, 23]. In this observational study, we prospectively recruited 70 patients with $\mathrm{mTBI}$ and followed up their cognitive functioning with functional and neuropsychological data for 1 year. In particular, 24 patients who completed all baseline and 5 follow-up sessions were selected for retrospective determination of the status of WM decline or recovery at each time point during the 1-year follow-up period. Although the relatively 
small dataset used in this study might be a concern, $\mathrm{k}$-fold cross-validation $(\mathrm{k}=10$ in our case) was applied to generate a reliable and unbiased estimate of machine learning model performance on a limited dataset sample [19]. Stated otherwise, we used a limited sample to estimate how the model is expected to perform in general when used to make predictions on unseen data. The systematic processing procedures and the results of this study thus prove the feasibility of using machine learning-based approaches to reveal predictive biomarkers related to poor postconcussive WM outcomes.

\subsection{Neuropsychological assessments are not predictive of postconcussion cognitive decline}

None of the baseline assessments of clinical neuropsychiatric symptoms (e.g. GOSE, sleep quality, depression and anxiety) or the self-reported PCS burden (RPQ) correlated significantly with WM changes between 6 weeks and 3 months, between 3 and 6 months, and between 6 months and 1 year after concussion in this study. These results substantiate the idea that the baseline neuropsychological assessments and PCS burden may not be predictive of postconcussive cognitive outcomes. Moreover, this was true in a previous study, which demonstrated that a lower cognitive reserve, but not a worse PCS diagnosis, was associated with a poor cognitive outcome following mTBI [24]. Studies have also shown that mTBI-induced differences in WM functional activity are observable; however, differences in neuropsychological and behavioral performance were not evident, suggesting that the deficits of WM functional activity estimated from fMRI may have higher sensitivity to long-term WM deficits in MTBI than to neuropsychological evaluations alone [13]. In addition, the time period between concussion and baseline assessment is also not related to the postconcussive WM changes, which indicates that the biomarkers identified in this study are not biased by the start time of the initial scan (within 1 week after mTBI in this research). Collectively, these results provide supportive evidence for using fMRI biomarkers elicited from baseline WM functional activity to predict long-term postconcussive cognitive outcomes, as done in our research.

\subsection{Age and sex effect in postconcussive working memory impairment}

Recent studies have indicated the importance of age and sex effect in the context of $\mathrm{mTBI}$, as elderlies and females are especially predisposed to postconcussive neurocognitive symptoms [25-28]. This is also true in our data, where age and sex factor showed significant contribution to the predictive models (Figure 3, Figure 4, Figure 5 and Figure 6), which possibly points to the age and sexual vulnerability factor to persistent postconcussive WM impairments. Further investigation is needed to corroborate the findings and to identify the mechanisms behind the involvement of age and sex in mTBI, especially in long-term postconcussive WM outcome.

\subsection{The role of WM task-induced deactivation regions in reflecting postconcussive cognitive decline}

In addition to considering WM functional activity as a potential fMRI biomarker for postconcussive WM decline, the WM task-induced default mode network (DMN) deactivation regions were considered in this research. The DMN, which is known to be active during rest and to deactivate during externally oriented tasks, may be essential for optimal WM operation [29]. The failure to deactivate the DMN during a cognitive task may limit the ability to reallocate cognitive resources for task execution [30,31]. Superior WM performance might be associated with the balance in the communication between taskpositive (activation) and task-negative (deactivation) regions in the context of effortful task execution [32]. Therefore, exclusively examining abnormalities in aberrant activation may be insufficient for a complete understanding of WM pathology [29]. Thus, we focused on both activation and deactivation deficits, marking a substantive advancement over prior works. In our work, approximately one-third to one-half of fMRI biomarkers exhibited deactivation deficits and significantly contributed to the prediction of postconcussive 
WM impairments. The results suggest that characterizing both activation and deactivation deficits is crucial for a complete understanding of WM dysfunction in mTBI.

\subsection{Scientific merit and clinical implications}

The systematic characterization of WM functional deficits may have crucial therapeutic implications in patients with postconcussive WM dysfunction, facilitating rehabilitation intervention planning in selected patients. Studies have suggested the potential application of brain functional activation and deactivation patterns in WM tasks for early neurocognitive training referral, training intensity planning, or even functional recovery prediction [13, 33]. Moreover, pharmacological interventions such as catecholaminergic treatment with methylphenidate improve cognitive performance in patients with severe TBI through the normalization of WM activation patterns [34]. Manktelow et al. demonstrated that compromised functional integrity and connectivity strength between key structures of the WM activation pattern in patients with TBI can be treated with methylphenidate to improve cognitive performance and that methylphenidate's pharmacologic effect may be more beneficial in patients with moderately severe cognitive deficits [35]. This treatment outcome correlation has not been well-explored for mTBI; nevertheless, our study results also indicate that the assessment of WM functional deficits may facilitate early rehabilitation interventions for patients with possible poor long-term cognitive performance and may thus reduce heterogeneity in treatment response and cognitive outcome. These fMRI predictive biomarkers exhibit potential to reflect the functional dynamics of neuroplasticity mechanisms in the injured brain. Our results support the hypothesis that pooled fMRI, demographic, and neuropsychological baseline biomarkers can satisfactorily predict postconcussive WM deficits during a 1-year period. Future studies must focus on using these predictive biomarkers as a patient stratification strategy to provide early intervention for patients who are at high risk of postconcussive WM dysfunctions.

\section{Conclusions}

This study examined postconcussion cognitive changes during a 1-year follow-up period. Consistent with a previous study [36], in our study, patients with mTBI demonstrated significant cognitive recovery at the third month after concussion, followed by worsened performance after 6 months, which persisted until 1 year after concussion (Figure 1). Approximately half of the patients experienced prolonged cognitive impairment, including impaired WM, DS, and AMT at 1-year follow up (Figure 2). The results are similar to those of previous studies, indicating that significant postconcussion cognitive impairment may persist for years despite some recovery over time [36]. Even a single concussion can lead to persistent cognitive impairment in approximately half of patients [10]. We constructed machine learning-based predictive models to differentiate patients at high risk for poor cognitive outcomes at the representative time periods after concussion. Satisfactory predictions were achieved for patients with mTBI whose cognitive function did not recover after 3 months (Figure 3), worsened at 6 months (Figure 4, did not recover at 1 year (Figure 5), and worsened at 1 year compared with baseline (Figure 6). This study demonstrated the feasibility of prediction individualization for long-term postconcussion cognitive outcomes by using pooled fMRI, demographic, and neuropsychological features and further suggests the possibility of early therapeutic intervention, such as neurocognitive training, for individuals with $\mathrm{mTBI}$ with poor long-term cognitive outcomes to reduce postconcussive cognitive decline and risk of chronicity [37]. fMRI may also be used to evaluate and guide treatment strategies, specifically targeting brain areas involved in postconcussive cognitive decline [13, 38, 39].

Supplementary Materials: The following are available online at www.mdpi.com/xxx/s1, Figure S1: N-back WM task diagram, Figure S2: Flow chart, Table S1: WM 2-back task-induced activation and 
deactivation peak regions among all participants, Table S2: Demographics, clinical and cognitive characteristics, and WM task performance of the participants.

Author Contributions: C.-Y. C. and Y.-H. C. designed the experiment; Y.-T. L., Y.-C. C., Y.-L. C., and D.-P. K. analyzed data; J.-J. C. and S. -H. T. edited manuscript; Y.-T. L., Y.-C. C., Y.-L. C., and D.-P. K. collected and interpreted the patient data; Y.-T. L. , Y.-C. C. and Y.-L. C. were major contributors in writing the manuscript. All authors read and approved the final script.

Funding: This research was funded by Ministry of Science and Technology, Taiwan (MOST1082321-B-038-008 and MOST110-2314-B-038-086-MY3) and Taipei Medical University, Taiwan (TMU109-AE1-B18).

Institutional Review Board Statement: This study has been reviewed and approved by the Institutional Review Board of Taipei Medical University Hospital before data collection (TMUH TMUJIRB No. 201504083, N201612008, and N201904032) and conducted according to the original and amended Declaration of Helsinki.

Informed Consent Statement: Informed consent was obtained from all participants involved in the study at Taipei Medical University Hospital.

Consent to Publication: All authors have read and approved the submitted manuscript. The manuscript has not been submitted elsewhere nor published elsewhere in whole or in part.

Availability of Data and Materials: Open-source software is available from the resources as cited, or from the authors on request. Other source codes generated in this study can be downloaded from https://github.com/YiTienLi/matlab-toolbox. The data are not publicly available due to the wording of the consent that participants gave to this project.

Competing Interests: The authors declare that they have no competing interests. The funders had no role in the design of the study; in the collection, analyses, or interpretation of data; in the writing of the manuscript, or in the decision to publish the results

Acknowledgments: This manuscript was edited by Wallace Academic Editing.

\section{References}

1. Vanderploeg, R.D., H.G. Belanger, and G. Curtiss, Mild traumatic brain injury and posttraumatic stress disorder and their associations with health symptoms. Archives of physical medicine and rehabilitation, 2009. 90(7): p. 1084-1093.

2. Rohling, M.L., et al., A meta-analysis of neuropsychological outcome after mild traumatic brain injury: re-analyses and reconsiderations of Binder et al. (1997), Frencham et al. (2005), and Pertab et al. (2009). Clin Neuropsychol, 2011. 25(4): p. 608-23.

3. Daneshvar, D.H., et al., Long-term consequences: effects on normal development profile after concussion. Phys Med Rehabil Clin N Am, 2011. 22(4): p. 683-700, ix.

4. Hall, R.C., R.C. Hall, and M.J. Chapman, Definition, diagnosis, and forensic implications of postconcussional syndrome. Psychosomatics, 2005. 46(3): p. 195-202

5. Blanchet, S., et al., Impact of divided attention during verbal learning in young adults following mild traumatic brain injury. Brain Inj, 2009. 23(2): p. 111-22.

6. Maddocks, D. and M. Saling, Neuropsychological deficits following concussion. Brain Inj, 1996. 10(2): p. 99-103.

7. Mangels, J.A., et al., Effects of divided attention on episodic memory in chronic traumatic brain injury: a function of severity and strategy. Neuropsychologia, 2002. 40(13): p. 2369-85.

8. Rutherford, W., J. Merrett, and J. McDonald, Symptoms at one year following concussion from minor head injuries. J Injury, 1979. 10(3): p. 225-230.

9. Sterr, A., et al., Are mild head injuries as mild as we think? Neurobehavioral concomitants of chronic post-concussion syndrome. J BMC neurology, 2006. 6(1): p. 1-10.

10. McInnes, K., et al., Mild Traumatic Brain Injury (mTBI) and chronic cognitive impairment: A scoping review. PLoS One, 2017. 12(4): p. e0174847.

11. Rabinowitz, A.R. and H.S. Levin, Cognitive sequelae of traumatic brain injury. Psychiatr Clin North Am, 2014. 37(1): p. 1-11.

12. McAllister, T.W., et al., Mechanisms of working memory dysfunction after mild and moderate TBI: evidence from functional MRI and neurogenetics. J Neurotrauma, 2006. 23(10): p. 1450-67.

13. Chen, C.J., et al., Working memory in patients with mild traumatic brain injury: functional MR imaging analysis. Radiology, 2012. 264(3): p. 844-51.

14. Wortzel, H.S. and D.B. Arciniegas, Treatment of post-traumatic cognitive impairments. Current treatment options in neurology, 2012. 14(5): p. 493-508

15. Cicerone, K.D., et al., Evidence-based cognitive rehabilitation: updated review of the literature from 2003 through 2008. Archives of physical medicine and rehabilitation, 2011. 92(4): p. 519-530. 
16. Owen, A.M., et al., N-back working memory paradigm: a meta-analysis of normative functional neuroimaging studies. Hum Brain Mapp, 2005. 25(1): p. 46-59.

17. Witt, S.T., M. Warntjes, and M. Engström, Increased fMRI sensitivity at equal data burden using averaged shifted echo acquisition. J Frontiers in neuroscience, 2016. 10: p. 544.

18. Luo, W.L. and T.E. Nichols, Diagnosis and exploration of massively univariate neuroimaging models. Neuroimage, 2003. 19(3): p. 1014-32.

19. Bengio, Y. and Y. Grandvalet, No unbiased estimator of the variance of k-fold cross-validation. Journal of machine learning research, 2004. 5(Sep): p. 1089-1105.

20. Cnossen, M.C., et al., Prediction of persistent post-concussion symptoms after mild traumatic brain injury. Journal of neurotrauma, 2018. 35(22): p. 2691-2698.

21. Langer, L.K., et al., Prediction of risk of prolonged post-concussion symptoms: Derivation and validation of the TRICORDRR (Toronto Rehabilitation Institute Concussion Outcome Determination and Rehab Recommendations) score. PLoS medicine, 2021. 18(7): p. e1003652.

22. Hellstrøm, T., et al., Predicting outcome 12 months after mild traumatic brain injury in patients admitted to a neurosurgery service. Frontiers in neurology, 2017. 8: p. 125.

23. Tenovuo, O., et al., Assessing the Severity of Traumatic Brain Injury-Time for a Change? Journal of clinical medicine, 2021. 10(1): p. 148.

24. Stenberg, J., et al., Cognitive reserve moderates cognitive outcome after mild traumatic brain injury. Archives of physical medicine and rehabilitation, 2020. 101(1): p. 72-80.

25. Bazarian, J.J., et al., Sex differences in outcome after mild traumatic brain injury. J Neurotrauma, 2010. 27(3): p. 527-39.

26. Hsu, H.L., et al., Sex Differences in Working Memory after Mild Traumatic Brain Injury: A Functional MR Imaging Study. Radiology, 2015. 276(3): p. 828-35.

27. Chen, D.Y., et al., Effect of Age on Working Memory Performance and Cerebral Activation after Mild Traumatic Brain Injury: A Functional MR Imaging Study. Radiology, 2016. 278(3): p. 854-62.

28. Levin, H.S., et al., Association of sex and age with mild traumatic brain injury-related symptoms: a TRACK-TBI study. JAMA network open, 2021. 4(4): p. e213046-e213046.

29. Anticevic, A., G. Repovs, and D.M. Barch, Working memory encoding and maintenance deficits in schizophrenia: neural evidence for activation and deactivation abnormalities. Schizophrenia bulletin, 2013. 39(1): p. 168-178.

30. $\mathrm{Hu}, \mathrm{Y}$. , et al., Resting-state glutamate and GABA concentrations predict task-induced deactivation in the default mode network. J Neurosci, 2013. 33(47): p. 18566-73.

31. Singh, K.D. and I.P. Fawcett, Transient and linearly graded deactivation of the human default-mode network by a visual detection task. Neuroimage, 2008. 41(1): p. 100-12.

32. Anticevic, A., et al., When less is more: TPJ and default network deactivation during encoding predicts working memory performance. Neuroimage, 2010. 49(3): p. 2638-2648.

33. Wylie, G.R., et al., Cognitive improvement after mild traumatic brain injury measured with functional neuroimaging during the acute period. PloS one, 2015. 10(5): p. e0126110.

34. Levin, H., et al., Methylphenidate treatment of cognitive dysfunction in adults after mild to moderate traumatic brain injury: rationale, efficacy, and neural mechanisms. Frontiers in neurology, 2019. 10: p. 925.

35. Manktelow, A.E., et al., Working memory after traumatic brain injury: the neural basis of improved performance with methylphenidate. Frontiers in behavioral neuroscience, 2017. 11: p. 58.

36. Carroll, E.L., et al., Mild traumatic brain injury recovery: a growth curve modelling analysis over 2 years. Journal of neurology, 2020. 267(11): p. 3223-3234.

37. Bohnen, N., J. Jolles, and A. Twijnstra, Neuropsychological deficits in patients with persistent symptoms six months after mild head injury. Neurosurgery, 1992. 30(5): p. 692-696.

38. Laatsch, L., et al., Investigating the neurobiological basis of cognitive rehabilitation therapy with fMRI. Brain Injury, 2004. 18(10): p. 957-974.

39. Strangman, G., et al., Functional neuroimaging and cognitive rehabilitation for people with traumatic brain injury. American journal of physical medicine \& rehabilitation, 2005. 84(1): p. 62-75. 